Patrick D. Converse \& Frederick L. Oswald (2014) Thinking Ahead: Assuming Linear Versus Nonlinear Personality-Criterion Relationships in Personnel Selection, Human Performance, 27:1, 61-79, DOI: 10.1080/08959285.2013.854367

\title{
Thinking Ahead: Assuming Linear Versus Nonlinear Personality-Criterion Relationships in Personnel Selection
}

\author{
Patrick D. Converse ${ }^{a}$ \\ Frederick L. Oswald ${ }^{b}$ \\ ${ }^{\text {a }}$ Florida Institute of Technology \\ ${ }^{\mathrm{b}}$ Rice University
}

Recent studies suggest that the form of some personality-performance relationships may be curvilinear, meaning that traditional top-down selection is inefficient in capitalizing on underlying personality-performance relationships. This study examines how mean performance is affected by how well the selection method is aligned with the nature of personality-criterion relationships. A simulation manipulated the linearity or nonlinear inflection point of predictorcriterion relationships, and several selection approaches were implemented that varied in level of congruence with these relationships. Results indicate that incongruence can produce notable decrements in mean performance under some conditions. Some evidence also suggests that decrements can be greater when linearity is assumed but relationships are nonlinear (vs. when nonlinearity is assumed but relationships are linear), selection ratios are smaller, and a single predictor is used. 
In personnel selection settings, predictor-criterion relationships are often best described by linear models. This has been most clearly demonstrated with ability-related constructs, given substantial large-sample evidence indicating the absence of nonlinear relationships (e.g., Coward $\&$ Sackett, 1990). However, growing evidence suggests that some relationships between personality traits and job performance may in fact have a curvilinear form. Research has recently demonstrated curvilinear relationships for both Conscientiousness and Emotional Stability with job performance, where positive relationships existed at lower levels of these characteristics, but these relationships gradually decreased at higher levels or even became negative for lower complexity jobs (Le, Oh, Robbins, Ilies, Holland, \&Westrick, 2010; see also LaHuis, Martin, \& Avis, 2005). This evidence suggests that at least in some situations involving personality, selection decisions might be more effectively based on a nonlinear approach. That is, certain characteristics may only be desirable up to a point, and in some cases high trait levels may in fact be detrimental to performance (e.g., employees who are too high in Conscientiousness may be too rigid or compulsive to function well on a job that requires some flexibility). Assuming these nonlinear relationships are stable, then selection procedures should be implemented in accordance with them in order to maximize validity and the benefits that stem from it.

However, we acknowledge the challenge of correctly identifying those situations calling for a nonlinear selection approach and, once identified, determining the precise nature of the curvilinear relationship that does not capitalize on chance. Theory has suggested curvilinear relationships (e.g., between Conscientiousness and task performance), and more complex and nonlinear theorizing and modeling has long been recommended in selection research (Dunnette, 1963); however, such theories are usually not precise enough to specify the exact location of the associated inflection point that would be critical to making appropriate selection decisions. Therefore, empirical evidence is essential to implement selection processes in situations where nonlinear relationships may hold. That said, empirical results related to this issue have been quite mixed. Furthermore, even if a given curvilinear personality-performance relationship is theoretically expected and reasonably well established, the inflection point may vary across jobs, criteria, and measures making it difficult to correctly implement a nonlinear selection approach without fairly extensive local research. Thus, when using personality characteristics as predictors, it may be quite common for selection procedures to be improperly specified to some extent, where (a) a linear approach is implemented when in fact nonlinearity holds, (b) a nonlinear approach is implemented when in fact linearity holds, or (c) a nonlinear approach is implemented but the inflection point is incorrectly identified.

This simulation study explores these issues more closely, focusing on performance decrements that are associated with incorrectly implementing personality-based selection procedures. As discussed in more detail later, Monte Carlo simulation is used to examine how mean job performance is affected when selection procedures are specified properly versus improperly in terms of linearity or the nonlinear inflection point. The goal was to begin uncovering the performance consequences associated with differing levels of alignment between the assumptions of the hiring organization and the actual personality-criterion relationships. 


\section{Personality in Personnel Selection}

Clearly, personality constructs are a major focus of selection research and practice (e.g., Hough \& Oswald, 2008; Oswald \& Hough, 2010; Sackett \& Lievens, 2008). Numerous metaanalyses have demonstrated that some personality characteristics demonstrate modest but consistent predictive validity across jobs (e.g., Barrick \& Mount, 1991; Barrick, Mount, \& Judge, 2001; Hurtz \& Donovan, 2000; Salgado, 1997). Most of these meta-analyses have focused on Big Five personality traits (Conscientiousness, Emotional Stability, Agreeableness, Extraversion, and Openness to Experience), with their results having built what is now viewed as a major tenet of organizational research: Conscientiousness tends to predict performance across a variety of criteria and jobs, and the other four personality traits predict some outcomes or success for some occupations, such as Openness predicting training performance (see Barrick et al., 2001). Furthermore, additional evidence suggests that when theory is employed to match personality constructs with criteria, then the validity for each of the Big Five measures increases (e.g., Hogan \& Holland, 2003; Oswald \& Hough, 2010). Thus, there is both theoretical and empirical support for the use of personality variables in selection.

\section{Personality-Criterion Relationships}

Despite meta-analytic and other research evidence in support of personality variables as linear predictors of performance, theory suggests that the linear form of these relationships is questionable. In contrast with cognitive ability, where numerous studies indicate positive linear relationships with performance across jobs (e.g., Coward \& Sackett, 1990; Schmidt \& Hunter, 1998), several researchers have posited that personality-performance relationships are likely to be more complex in terms of form and even direction (e.g., Murphy, 1996; Ones, Dilchert, Viswesvaran, \& Judge, 2007; Tett \& Christiansen, 2007). Both conceptual considerations and empirical evidence are somewhat mixed regarding the linearity of personality-performance relationships, suggesting the form of these relationships may be more complex, vary across traits and situations, and require greater statistical power to detect. In particular, previous arguments and evidence suggest personality-performance relationships may be (a) linear, where higher personality levels are consistently associated with higher performance levels; (b) asymptotic, where higher personality levels are associated with higher performance levels until an asymptote is reached, at which point higher personality levels are associated with the same performance level; or (c) quadratic, where higher personality levels are associated with higher performance levels until an inflection point is reached, at which point higher personality levels are associated with lower performance levels (note that after the inflection point, performance levels can still be relatively high - e.g., well above mean performance - although they will be lower than performance at the inflection point). 


\section{Conceptual Considerations}

The notion of nonlinear relationships between psychological variables appears in several areas of research (see Grant \& Schwartz, 2011). Some arguments suggest that personalityperformance relationships in particular may often be curvilinear. For example, Conscientiousness involves being dependable and achievement oriented; thus, Conscientiousness is typically found to be beneficial for performance across most jobs (e.g., Barrick \& Mount, 1991; Barrick et al., 2001). However, some have argued that in some cases in the workplace, higher levels of Conscientiousness may in fact be maladaptive. For instance, Robie and Ryan (1999) suggested that individuals with moderate levels of Conscientiousness may have higher job performance, because those with very low levels may be disorganized or unmotivated, whereas those with very high levels may be inflexible or perfectionistic. Although these are handicaps for most jobs, rigidity and perfectionism might be especially problematic in jobs that often call upon flexibility and creativity (e.g., managers, poets). Similar arguments could be made for other Big Five characteristics such as Emotional Stability and Agreeableness. Emotional Stability may tend to be adaptive in work settings (e.g., Salgado, 1997), but in some cases a moderate level of anxiety (and thus a moderate level of Emotional Stability) may produce better performance as a result of its implications for heightened attention and motivation (see Le et al., 2010; Nettle, 2006). This notion is aligned with the classic Yerkes-Dodson Law (Yerkes \& Dodson, 1908) that portrays the relationship between arousal and performance as an inverted U. Moderate levels of Agreeableness may also lead to higher performance in some contexts, such as in customer relations, where very high or very low levels may result in ineffective interactions (Arthur, Woehr, \& Graziano, 2001).

On the other hand, in many situations personality-performance relationships may be linear. In more complex and demanding jobs (e.g., scientist), for instance, high levels of Conscientiousness (e.g., being disciplined, goal focused, and detail oriented) may be necessary rather than maladaptive (Le et al., 2010). Furthermore, even if some true curvilinearity exists in a broad population of individuals, the relationships may be effectively linear for the purposes of selecting from a narrower population of job applicants. For example, some arguments supporting the notion of curvilinear relationships seem to focus on clinical levels of the personality traits at the extremes (e.g., being completely disorganized or excessively rigid) and how those characteristics would be maladaptive for life functioning, let alone work performance (Markon, Krueger, \& Watson, 2005). In this case, nonlinearity would apply to very few people in the workplace, and any nonlinearity that exists at that level may be impractical to model and incorporate into a selection system using measures developed for selection settings. In addition, even when curvilinearity exists within the normal personality range assessed by a selection tool, a high inflection point (e.g., 3 SDs above the mean) would mean the relationship remains positive up to some high level on the personality characteristic continuum and thus traditional selection approaches that assume linearity (e.g., top-down selection) could still be reasonably effective. This point is consistent with Dawes and Corrigan's (1974) conclusion that "linear 
models are good approximations to all multivariate models that are conditionally monotone in each predictor variable" (p. 98). In sum, there appear to be reasonable conceptual arguments that support both linear and nonlinear relationships between personality and organizational outcomes such as job performance.

\section{Empirical Evidence}

Consistent with these conceptual considerations, empirical evidence related to this issue is also mixed. A few studies have reported meaningful nonlinear relationships in their data. Day and Silverman (1989) reported curvilinear relationships between impulse expression and ratings of timeliness of work and cooperation in a sample of accountants, where moderate scorers had better performance ratings and low and high scorers had worse ratings. LaHuis et al. (2005) found evidence of nonlinear Conscientiousness-performance relationships in two independent samples of clerical employees. These researchers predicted an asymptotic relation, with a positive relationship in the low to moderate range but no relationship in the moderate to high range, and found a slight inverted $U$ relationship (Study 1) and an asymptotic relationship (Study 2). Benson and Campbell (2007) predicted inverted U relationships between derailing/dark side personality variables and leadership performance. Results from two samples of managers suggested asymptotic curves (Study 1) and inverted U curves (Study 2). Le et al. (2010) also expected curvilinear personality-performance relationships, where these relationships would disappear at higher levels of personality. These researchers found nonlinear relationships between two personality variables (Conscientiousness and Emotional Stability) and three performance dimensions (task performance, organizational citizenship behavior, and counterproductive work behavior) in a sample of individuals from a variety of jobs, with the shape of the relationship depending on the variables involved and job complexity (Study 1). Whetzel, McDaniel, Yost, and Kim (2010) explored for linear, quadratic, and cubic relationships between personality and job performance using the 32 Occupational Personality Questionnaire scales in a sample of professionals in a financial services firm. Results indicated a quadratic relationship (inverted $\mathrm{U}$ ) for Conscientiousness given a $\Delta \mathrm{R} \geq .05$ criterion; a few other scales demonstrated quadratic or cubic relationships given a $\Delta \mathrm{R} \geq .025$ or .01 criterion. Finally, a few other studies focusing on other outcomes have also found curvilinear relationships. Cucina and Vasilopoulos (2005), for example, found evidence of nonlinear relationships between Conscientiousness (inverted U shaped) and Openness to Experience (U shaped) and grade point average. Vasilopoulos, Cucina, and Hunter (2007) also found evidence of quadratic relationships for Conscientiousness and Emotional Stability predicting training performance.

In contrast, other studies have not supported nonlinear relationships. Robie and Ryan (1999) explored for nonlinear Conscientiousness-performance relationships using quadratic and cubic models. They found no evidence of nonlinear relationships across five independent work samples (Ns ranged from 146 to 999): federal government employees, multiorganizational private sector employees, Department of Defense managers, wholesale sales representatives, and 
long-haul semitruck drivers. These researchers concluded that "in most instances, practitioners probably do not need to be concerned that if they choose to adopt a top-down strategy of selection with a measure of conscientiousness, that they will somehow choose an individual who will perform poorly on the job" (p. 167). In addition, other studies that have obtained some support for nonlinear relationships have also reported results that do not support these relationships. For example, despite positive results in Study 1, Le et al. (2010) did not find curvilinear relationships between Conscientiousness and the three performance dimensions examined or between Emotional Stability and task performance in their Study 2. In addition, although Day and Silverman (1989) found curvilinear relationships between impulse expression and two outcome measures, they did not report nonlinear results for four other criterion measures (e.g., potential for success, client relations). Similarly, Whetzel et al. (2010) did not find evidence for nonlinear relationships for 31 to 25 (depending on $\Delta \mathrm{R}$ criterion) out of 32 personality scales. Thus, empirical results related to linearity are fairly inconclusive.

Finally, an additional complication in this context is that Le et al. (2010) recently found that even when curvilinear relationships hold, the associated inflection points tended to be lower for lower complexity jobs than for higher complexity jobs, suggesting that lower complexity jobs place lower demands on personality as well as ability characteristics. This indicates that the location of the inflection point - a critical piece of information for effectively implementing selection procedures in situations involving curvilinear relationships - may vary substantially depending on the job context. In fact, Le et al. (2010) reported inflection points ranging from 0.10 to 3.97 in Z-score form.

\section{Selection Practice}

Despite theory and research suggesting nonlinear relationships between personality and performance, the relevant conditions and findings are often complex, conditional, and not precisely measured, making it difficult at this point to determine what specific approach or inflection point to use in selection systems involving personality measures. In addition, the regression weight for the squared predictor of a linear regression can be imprecise if the variable itself is unreliable (see Shepperd, 1991), and this in turn would adversely affect the precision in estimating the inflection point of a nonlinear relationship. Therefore, even if selection specialists generally suspect a nonlinear relationship in a given situation, it may be quite difficult to identify the specific inflection point without conducting additional research efforts on large samples that are specific to the setting of interest. Given the expenses associated with conducting this type of research without the guarantee of offsetting rewards, and the general insensitivity to the possibility of nonlinear relationships, it perhaps is understandable why selection in organizations often proceeds under the assumption of linearity. This then means there is substantial opportunity for a lack of fit between the personality-related selection systems that are implemented and the underlying personality-performance relationships. 
In current selection practice involving personality measures, the traditional and straightforward approach of selecting top-down on a given variable or on a linear composite of multiple variables is consistent with the assumption of linear relationships. In contrast, a nonlinear assumption is implied in profile matching: identify an ideal personality profile for a given job, quantify how close the ideal profile is to the profile of each applicant's scores in terms of similar patterns or levels, then use this quantity to make selection decisions. Unless profile matching assumes an ideal profile with very high or very low levels of each personality characteristic, curvilinear relationships are assumed at least implicitly, because it suggests that performance should reach a maximum somewhere in the midrange (moderately low to moderately high) for at least some personality traits that are involved (see Whetzel et al., 2010).

\section{Current Study}

When taken all together, the considerations we have examined to this point suggest that selection decisions involving personality variables might be improved by examining nonlinear personality-performance relationships that may exist. The current Monte Carlo simulation across multiple personnel selection scenarios addresses the extent to which mean performance resulting from selection is negatively affected by misalignment between personality-performance relationships and the models that drive selection decisions. Both linear and nonlinear personality-performance relationships are simulated to explore the consequences of correctly versus incorrectly specifying both the form of the relationship (linear vs. nonlinear) and the location of the inflection (turning) point in nonlinear relationships, both of which are likely to be critical for properly implementing more effective selection systems involving personality variables. Simulations allow us to reveal the extent that increased selection system accuracies might allow organizations to reap more benefit out of personality predictors (or other predictors) that demonstrate nonlinear relationships with criteria.

\section{Method}

The simulation was developed using SAS/IML and involved seven major steps. First, the simulation generated data based on two predictor variables representing Conscientiousness and Emotional Stability given their intercorrelation and a sample size considered to be large in some selection research settings $(\mathrm{N}=200)$. For each predictor, the simulation generated scores that were uncorrelated and distributed standard normal $(\mathrm{M}=0, \mathrm{SD}=1)$. Then these variables were transformed to reproduce the desired target correlation within the appropriate bounds of sampling error variance; the target correlation is from van der Linden, Nijenhuis, and Bakker's (2010) uncorrected meta-analytic estimate (see Table 1). 
TABLE 1 Simulation Correlation Matrix Between Predictors and Criterion

\begin{tabular}{|c|c|c|c|}
\hline & 1 & 2 & 3 \\
\hline \multicolumn{4}{|l|}{ Predictors } \\
\hline 1. Conscientiousness & - & & \\
\hline 2. Emotional Stability & .32 & - & \\
\hline \multicolumn{4}{|l|}{ Criterion } \\
\hline 3. Criterion (Performance) & $r_{\text {con-perf }}$ & .14 & - \\
\hline
\end{tabular}

Second, criterion-related validities were then added to the target correlation matrix (see Table 1). Conscientiousness validity was set to $.10, .20$, or .30 to examine a range of potential selection situations such as entry-level, managerial, and academic contexts (e.g., Connelly \& Ones, 2010; Hurtz \& Donovan, 2000; Judge, Bono, Ilies, \& Gerhardt, 2002; Oh, Wang, \& Mount, 2011). Emotional Stability validity was set to .14 as a representative validity value (e.g., Connelly \& Ones, 2010; Hurtz \& Donovan, 2000; Judge et al., 2002; Oh et al., 2011).

Third, the simulation estimated the regression coefficients using this correlation matrix. These coefficients were then applied to the predictor data generated in the first step, and an additional random normal variable was generated to create prediction error variance, and then all three variables (two predictors and the error variance) were summed to create a job performance variable. This process thus simulated a scenario in which both predictor-criterion relationships were linear. The process was then repeated to reflect situations in which the Conscientiousnesscriterion relationship was curvilinear, with inflection points of 2.0, 1.5, 1.0, or 0.5 in standard score form (see Table 2). To be conservative in the number of nonlinear relationships involved and keep the complexity of the simulation manageable, Emotional Stability was modeled to have a linear relationship with the criterion in all conditions. These inflection point locations were chosen as representative values for situations where, in terms of validity, above-average Conscientiousness levels are desirable but only up to the inflection point (see Le et al., 2010; see Figure 1 for an illustration of these types of relationships). Each curvilinear relationship was introduced into the simulation regression model by adding squared Conscientiousness scores as a term. Note that this nonlinearity is thus added to the linear relationships based on past research; those validities capture the linear trends found in past data, even when the actual data show nonlinearity, and the additional squared term captures additional nonlinearity that can increase the overall model $\mathrm{R}^{2}$ in a regression analysis (see Table 3 for $\mathrm{R}^{2}$ estimates for the linear and nonlinear terms). This point is important for the literature in general, not just this simulation, namely, that if nonlinearities existed in past data and were modeled, that would have increased the model $\mathrm{R}^{2}$ and might have led to a different approach for personnel selection.

TABLE 2 Linearity and Inflection Points for Predictor-Criterion Relationships: Simulation Conditions

\begin{tabular}{|c|c|c|c|}
\hline \multicolumn{4}{|c|}{ Predictor-Criterion Relationship Form and Inflection Point } \\
\hline Criterion Variable & Conscientiousness & Emotional Stability & Variable Label \\
\hline 1 & Linear & Linear & IP: None \\
\hline 2 & $\mathrm{IP}=2.0$ & Linear & IP 2.0: C \\
\hline 3 & $\mathrm{IP}=1.5$ & Linear & IP $1.5: \mathrm{C}$ \\
\hline 4 & $\mathrm{IP}=1.0$ & Linear & IP $1.0: \mathrm{C}$ \\
\hline 5 & $\mathrm{IP}=0.5$ & Linear & IP $0.5: \mathrm{C}$ \\
\hline
\end{tabular}


FIGURE 1 Illustration of personality-criterion relationships. Note. IP = inflection point.

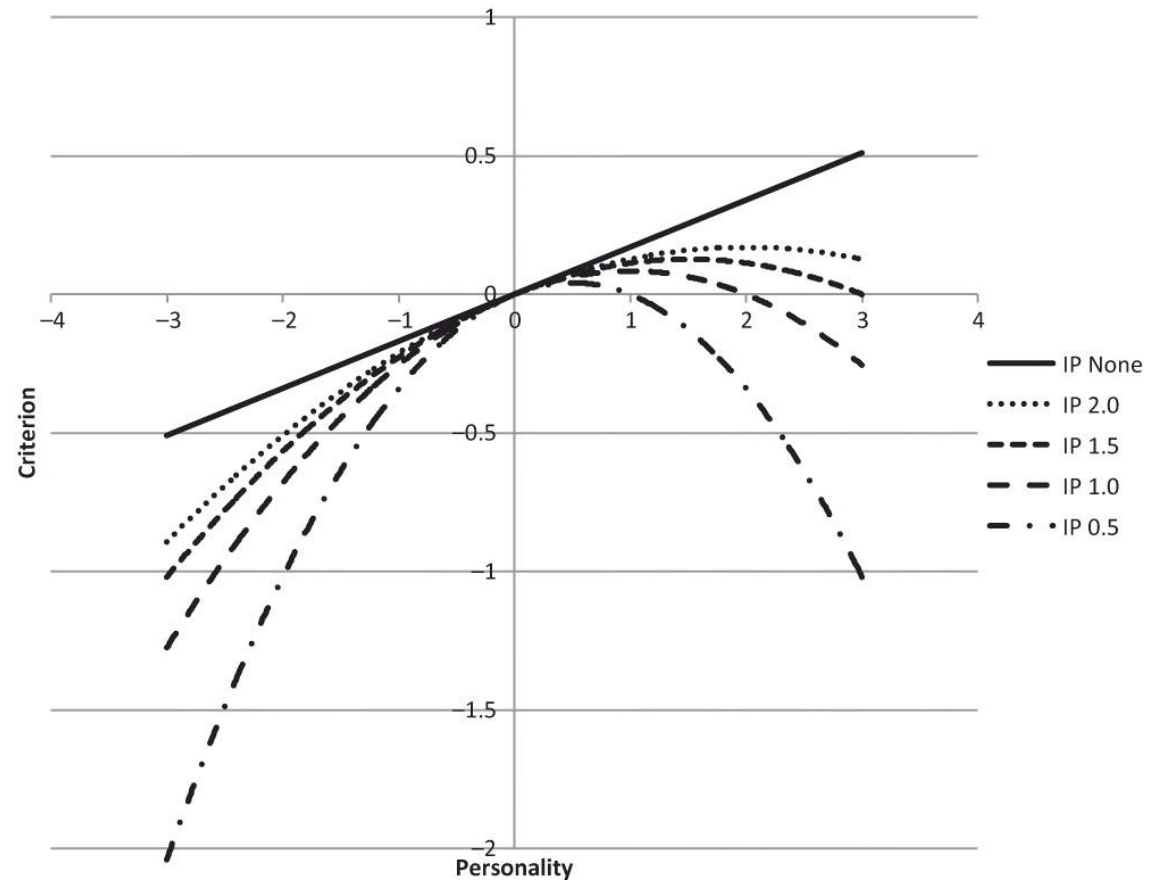

\begin{tabular}{|c|c|c|c|c|c|}
\hline & \multicolumn{5}{|c|}{$\Delta R^{2}$} \\
\hline & \multicolumn{5}{|c|}{ Predictor-Criterion Relationships } \\
\hline & IP: None & IP 2.0: $\mathrm{C}$ & IP 1.5: C & IP 1.0: C & IP 0.5: $\mathrm{C}$ \\
\hline \multicolumn{6}{|l|}{$\mathrm{C}$ Validity $=.10$} \\
\hline Step 1: C and ES & .021 & .020 & .020 & .020 & .020 \\
\hline Step 2: $\mathrm{C}^{2}$ & .000 & .001 & .001 & .003 & .009 \\
\hline \multicolumn{6}{|l|}{$\mathrm{C}$ Validity $=.20$} \\
\hline Step 1: $\mathrm{C}$ and ES & .043 & .043 & .043 & .042 & .040 \\
\hline Step 2: $\mathrm{C}^{2}$ & .000 & .004 & .006 & .014 & .055 \\
\hline \multicolumn{6}{|l|}{$\mathrm{C}$ Validity $=.30$} \\
\hline Step 1: C and ES & .093 & .092 & .091 & .089 & .080 \\
\hline Step 2: $\mathrm{C}^{2}$ & .000 & .007 & .014 & .033 & .128 \\
\hline
\end{tabular}

The inflection point (IP) and regression formula were manipulated by using the following equation (see Aiken \& West, 1991):

$\mathrm{IP}=-b_{1} / 2 b_{2}$,

where $b_{1}$ is the regression coefficient associated with the first-order term $(X)$ and $b_{2}$ is the regression coefficient associated with the squared term $\left(X^{2}\right)$. Thus, given the estimated value for $b_{1}$ and desired inflection point that were previously described, Equation 1 allows the simulation to calculate the corresponding value of $b_{2}$ to obtain curvilinear Conscientiousness-criterion relationships with specified inflection points. 
Fourth, the simulation created selection variables for use in making selection decisions. Two sets of selection variables were created: The first involved only Conscientiousness (to explore situations involving one predictor) and the second involved Conscientiousness plus Emotional Stability (to explore situations involving combining multiple predictors). To be consistent with the nature of the predictor-criterion relationships that were modeled, each set of selection variables consisted of five different selection variables (resulting in 10 selection variables total). Specifically, paralleling the criterion variables, selection variables were created under the assumption that (a) Conscientiousness had a linear relationship with the criterion or (b) Conscientiousness had a curvilinear relationship with the criterion with an inflection point of 2.0 1.5, 1.0, or 0.5 in standard score form. For Conscientiousness alone selection, selection variable operationalizations were based on these assumptions; for Conscientiousness plus Emotional Stability selection, selection variable operationalizations were based on these assumptions plus the assumption that Emotional Stability was a valid linear predictor.

All together, these different selection variables reflect different assumptions on the part of the hiring organization regarding the predictor-criterion relationships, with the nonlinear assumptions reflecting a forward-looking organization that considers new approaches to personnel selection. For instance, the hiring organization might assume two linear relationships and thus use a simple unit-weighted composite in selection, or it might assume one of the predictors has a curvilinear relationship with the criterion, such that predictors are rescored so that the highest scores are at the inflection point, and a greater distance from the assumed inflection point means a lower score for that predictor. The selection variables therefore involved ordinary predictor scores (when a linear personality-criterion relationship was assumed) and distances from an inflection point (when a nonlinear personality-criterion relationship was assumed). Thus, to create these variables, absolute distances from the assumed inflection points were first computed for conditions in which a curvilinear relationship was assumed such that greater distances from the inflection point (above or below) meant lower scores (see Table 4 for more details). Once the appropriate distance scores were computed, all variables (i.e., distances from inflection points and/or raw predictor scores) were standardized. For Conscientiousness alone selection, these Conscientiousness or Conscientiousness distance scores were used in making selection decisions; for Conscientiousness plus Emotional Stability selection, these Conscientiousness or Conscientiousness distance scores and Emotional Stability scores were summed to create selection composites that were used in making selection decisions. Note that this approach is generally consistent with Le et al.'s (2010) suggestion of using a double cutoff strategy involving both lower and upper predictor limits, in that both low and high scores decrease the probability of selection when curvilinear relationships are assumed. However, other operational approaches could be used such as computing predicted criterion scores for applicants using an equation that includes both linear and quadratic terms. 
TABLE 4 Selection Variable Calculation Across Simulation Conditions

\begin{tabular}{|c|c|c|}
\hline Selection Variable & Variable Calculation & Variable Label \\
\hline \multicolumn{3}{|c|}{ Selection approach: Conscientiousness alone } \\
\hline 1 & c & Linear \\
\hline 2 & $\left.(\operatorname{ABS}(2.0-C))^{*}-1\right)$ & D from 2.0: C \\
\hline 3 & $(\operatorname{ABS}(1.5-C)) *-1)$ & D from 1.5: C \\
\hline 4 & $\left.(\operatorname{ABS}(1.0-C))^{*}-1\right)$ & D from 1.0: C \\
\hline 5 & $\left.(\operatorname{ABS}(0.5-C))^{*}-1\right)$ & D from $0.5: \mathrm{C}$ \\
\hline \multicolumn{3}{|c|}{ Selection approach: Conscientiousness plus Emotional Stability } \\
\hline 6 & $C+E S$ & Linear \\
\hline 7 & $\left.(A B S(2.0-C))^{*}-1\right)+E S$ & D from 2.0: C \\
\hline 8 & $\left.(\operatorname{ABS}(1.5-\mathrm{C}))^{*}-1\right)+\mathrm{ES}$ & D from 1.5: C \\
\hline 9 & $\left.(\operatorname{ABS}(1.0-\mathrm{C}))^{*}-1\right)+\mathrm{ES}$ & D from 1.0: C \\
\hline 10 & $\left.(\operatorname{ABS}(0.5-C))^{*}-1\right)+E S$ & D from $0.5: \mathrm{C}$ \\
\hline
\end{tabular}

Fifth, for each selection condition, simulated job applicants were rank ordered on each of those selection variables, and selection decisions were made top-down according to the specified selection ratio (.10 or .50). Thus, this selection process was carried out 10 separate times, using one of the 10 selection variables each time. In other words, 10 different selection scenarios were simulated, reflecting different assumptions on the part of the hiring organization regarding predictors, linearity, and inflection points. Simulating these different scenarios allowed for an examination of the mean performance consequences associated with varying degrees of alignment between the assumptions of the hiring organization and the actual underlying predictor-performance relationships.

Sixth, mean performance across those individuals selected in the previous step was computed. Thus, this was carried out for each of the 10 selection variable scenarios and the two selection ratios.

Finally, to determine the distribution of our results for sample sizes of $\mathrm{N}=200$, this entire process (i.e., the six major steps just described) was replicated 1,000 times, and means across these replications were computed and are reported.

\section{Results}

Tables 5, 6, and 7 present mean performance for selected individuals (with performance scores in standard score form) when Conscientiousness criterion-related validity is .10, .20, and .30 , respectively. In these tables, each row represents a different selection approach based on different assumptions on the part of the hiring organization regarding personality-criterion relationships. For example, the first row of results for Conscientiousness alone selection ("Linear") reflects selection using ordinary Conscientiousness scores, and the first row of results for Conscientiousness plus Emotional Stability selection (also "Linear") reflects selection using a unit-weighted composite of ordinary Conscientiousness and Emotional Stability scores; in both cases, the assumption is that predictor-criterion relationships are linear. In contrast, the second row of results for Conscientiousness alone selection ("D from 2.0: C") reflects selection using Conscientiousness distance from 2.0, and the first row of results for Conscientiousness plus Emotional Stability (also "D from 2.0: C") reflects selection using a composite of Conscientiousness distance from 2.0 plus Emotional Stability; in both cases, the assumption is that the Conscientiousness-criterion relationship is curvilinear with an inflection point of 2.0 (with the additional assumption of a linear Emotional Stability-criterion relationship in the second case; see Table 4 for variable label explanations). Also in these tables, each column represents a different set of true underlying personality-criterion relationships. For example, the 
first column of results (IP: None) reflects a condition where both predictor-criterion relationships are linear, whereas the second column of results (IP 2.0: C) reflects a condition where the Conscientiousness-criterion relationship is nonlinear with an inflection point at 2.0 and the Emotional Stability-criterion relationship is linear (see Table 2 for variable label explanations). Thus, the tables show mean performance when selection variables are created in a way that follows the functional form of the predictor-criterion relationships (i.e., entries on the main diagonal within each selection ratio, in boldface) versus when the selection variable and the functional form are mismatched (i.e., all off-diagonal entries within each selection ratio).

\begin{tabular}{|c|c|c|c|c|c|}
\hline \multirow[b]{2}{*}{ Selection Operationalization } & \multicolumn{5}{|c|}{ Personality-Criterion Relationships } \\
\hline & IP: None & IP 2.0: C & IP 1.5: C & IP 1.0: C & IP 0.5: C \\
\hline \multicolumn{6}{|c|}{ Selection approach: Conscientiousness alone } \\
\hline \multicolumn{6}{|l|}{ Selection ratio $=.10$} \\
\hline Linear & .174 & .141 & .130 & .107 & .041 \\
\hline D from 2.0: C & .167 & .137 & .127 & .108 & .049 \\
\hline D from 1.5: C & .145 & .129 & .124 & .113 & .080 \\
\hline D from 1.0: C & .090 & .090 & .090 & .090 & .091 \\
\hline D from $0.5: \mathrm{C}$ & .042 & .053 & .057 & .064 & .086 \\
\hline \multicolumn{6}{|l|}{ Selection ratio $=.50$} \\
\hline Linear & .079 & .079 & .079 & .079 & .079 \\
\hline D from 2.0: C & .080 & .079 & .079 & .079 & .079 \\
\hline D from $1.5: \mathrm{C}$ & .079 & .079 & .079 & .079 & .080 \\
\hline D from 1.0: C & .070 & .074 & .075 & .077 & .084 \\
\hline D from $0.5: \mathrm{C}$ & .042 & .052 & .055 & .062 & 081 \\
\hline \multicolumn{6}{|c|}{ Selection approach: Conscientiousness plus Emotional Stability } \\
\hline \multicolumn{6}{|l|}{ Selection ratio $=.10$} \\
\hline Linear & .255 & .233 & .225 & .211 & .166 \\
\hline D from 2.0: C & .254 & .236 & .229 & .217 & .180 \\
\hline D from 1.5: C & .245 & .234 & .230 & .223 & .200 \\
\hline D from 1.0: C & .225 & .224 & .224 & .223 & .221 \\
\hline D from $0.5: C$ & .213 & .221 & .224 & .229 & .245 \\
\hline \multicolumn{6}{|l|}{ Selection ratio $=.50$} \\
\hline Linear & .116 & .116 & .116 & .116 & .116 \\
\hline D from 2.0: C & .116 & .116 & .116 & .116 & .116 \\
\hline D from 1.5: C & .116 & .116 & .116 & .117 & .117 \\
\hline D from 1.0: C & .113 & .115 & .116 & .117 & .121 \\
\hline$D$ from $0.5: C$ & .100 & .106 & .109 & .113 & .125 \\
\hline
\end{tabular}

Note. Personality-Criterion Relationships refers to the linearity of the Conscientiousness-criterion relationship, where IP: None indicates a linear relationship and IP 2.0: C, IP 1.5: C, IP 1.0: C, and IP 0.5: C indicate curvilinear relationships with IP = 2.0, 1.5, 1.0, and 0.5, respectively (the Emotional Stability-criterion relationship is always linear; see Table 2). Selection Operationalization refers to how Conscientiousness scores are used in selecting individuals, where Linear uses the Conscientiousness score itself and D from 2.0: C, D from 1.5: C, D from 1.0: C, and D from 0.5: $\mathrm{C}$ use the distance between the Conscientiousness score and 2.0, 1.5, 1.0, and 0.5, respectively. For Conscientiousness alone selection, these values alone are used; for Conscientiousness plus Emotional Stability selection, these values plus Emotional Stability scores are used (see Table 4). Within each selection ratio, bold indicates where the selection operationalization and the criterion are conceptually aligned and underline indicates the selection operationalization that yields maximum mean performance for each underlying predictor-criterion relationship. IP $=$ inflection point; $\mathrm{D}=$ distance $\mathrm{C}=$ Conscientiousness. 
TABLE 6 Mean Performance for Selected Individuals: Conscientiousness Validity $=.20$

\begin{tabular}{|c|c|c|c|c|c|}
\hline \multirow[b]{2}{*}{ Selection Operationalization } & \multicolumn{5}{|c|}{ Personality-Criterion Relationships } \\
\hline & IP: None & IP 2.0: C & IP 1.5: $\mathrm{C}$ & IP 1.0: $\mathrm{C}$ & IP 0.5: $\mathrm{C}$ \\
\hline \multicolumn{6}{|c|}{ Selection approach: Conscientiousness alone } \\
\hline \multicolumn{6}{|l|}{ Selection ratio $=.10$} \\
\hline Linear & .342 & .249 & .217 & .155 & -.030 \\
\hline D from 2.0: C & .334 & .251 & .223 & .167 & .001 \\
\hline D from 1.5: C & .284 & .238 & .223 & .192 & .099 \\
\hline D from 1.0: C & .192 & .192 & .192 & .192 & .189 \\
\hline D from $0.5: \mathrm{C}$ & .105 & .136 & .147 & .167 & .225 \\
\hline \multicolumn{6}{|l|}{ Selection ratio $=.50$} \\
\hline Linear & .153 & .153 & .153 & .152 & .149 \\
\hline D from 2.0: C & .153 & .153 & .153 & .152 & .149 \\
\hline D from 1.5: C & .152 & .153 & .153 & .153 & .152 \\
\hline D from 1.0: C & .134 & .144 & .147 & .153 & .169 \\
\hline D from $0.5: \mathrm{C}$ & .080 & .108 & .117 & .135 & .186 \\
\hline \multicolumn{6}{|c|}{ Selection approach: Conscientiousness plus Emotional Stability } \\
\hline \multicolumn{6}{|l|}{ Selection ratio $=.10$} \\
\hline Linear & .344 & .282 & .262 & .220 & .096 \\
\hline D from 2.0: C & .335 & .283 & .266 & .231 & .127 \\
\hline D from 1.5: C & .319 & .288 & .277 & .256 & .191 \\
\hline D from 1.0: C & .268 & .266 & .265 & .262 & .253 \\
\hline D from 0.5: C & .201 & .224 & .232 & .246 & .287 \\
\hline \multicolumn{6}{|l|}{ Selection ratio $=.50$} \\
\hline Linear & .162 & .162 & .162 & .161 & .158 \\
\hline D from 2.0: C & .162 & .162 & .162 & .161 & .158 \\
\hline D from 1.5: C & .163 & .163 & .164 & .164 & .162 \\
\hline D from 1.0: C & .155 & .161 & .162 & .166 & .174 \\
\hline D from 0.5: C & .121 & .139 & .145 & .157 & .189 \\
\hline
\end{tabular}

Note. Personality-Criterion Relationships refers to the linearity of the Conscientiousness-criterion relationship, where IP: None indicates a linear relationship and IP 2.0: C, IP 1.5: C, IP 1.0: C, and IP 0.5: C indicate curvilinear relationships with IP = 2.0, 1.5, 1.0, and 0.5 , respectively (the Emotional Stability-criterion relationship is always linear; see Table 2). Selection Operationalization refers to how Conscientiousness scores are used in selecting individuals, where Linear uses the Conscientiousness score itself and D from 2.0: C, D from 1.5: C, D from 1.0: C, and D from 0.5: $\mathrm{C}$ use the distance between the Conscientiousness score and 2.0, 1.5, 1.0, and 0.5, respectively. For Conscientiousness alone selection, these values alone are used; for Conscientiousness plus Emotional Stability selection, these values plus Emotional Stability scores are used (see Table 4). Within each selection ratio, bold indicates where the selection operationalization and the criterion are conceptually aligned and underline indicates the selection operationalization that yields maximum mean performance for each underlying predictor-criterion relationship. IP $=$ inflection point; $\mathrm{D}=$ distance; $\mathrm{C}=$ Conscientiousness.

TABLE 7 Mean Performance for Selected Individuals: Conscientiousness Validity $=.30$

\begin{tabular}{|c|c|c|c|c|c|}
\hline \multirow[b]{2}{*}{ Selection Operationalization } & \multicolumn{5}{|c|}{ Personality-Criterion Relationships } \\
\hline & IP: None & IP 2.0: C & IP 1.5: C & IP 1.0: C & IP 0.5: $\mathrm{C}$ \\
\hline \multicolumn{6}{|c|}{ Selection approach: Conscientiousness alone } \\
\hline \multicolumn{6}{|l|}{ Selection ratio $=.10$} \\
\hline Linear & .515 & .357 & .305 & .200 & -.099 \\
\hline D from 2.0: C & .499 & .359 & .312 & .218 & -.051 \\
\hline D from 1.5: C & .416 & .339 & .312 & .260 & .105 \\
\hline D from 1.0: C & .291 & .291 & .290 & .288 & .275 \\
\hline D from 0.5: C & .151 & .202 & .219 & .251 & .335 \\
\hline \multicolumn{6}{|l|}{ Selection ratio $=.50$} \\
\hline Linear & .235 & .234 & .234 & .231 & .219 \\
\hline D from 2.0: C & .235 & .234 & .234 & .231 & .220 \\
\hline D from 1.5: C & .233 & .234 & .234 & .233 & .224 \\
\hline D from 1.0: C & .206 & .222 & .227 & .236 & .255 \\
\hline D from 0.5: C & .123 & .169 & .183 & .211 & .286 \\
\hline \multicolumn{6}{|c|}{ Selection approach: Conscientiousness plus Emotional Stability } \\
\hline \multicolumn{6}{|l|}{ Selection ratio $=.10$} \\
\hline Linear & .470 & .367 & .332 & .262 & .059 \\
\hline D from 2.0: C & .451 & .365 & .336 & .278 & .107 \\
\hline D from 1.5: C & .407 & .355 & .337 & .300 & 191 \\
\hline D from 1.0: C & .331 & .325 & .323 & .317 & .293 \\
\hline D from $0.5: C$ & .225 & .262 & .274 & .297 & .353 \\
\hline \multicolumn{6}{|l|}{ Selection ratio $=.50$} \\
\hline Linear & .216 & .215 & .214 & .212 & .201 \\
\hline D from 2.0: C & .216 & .216 & .215 & .213 & .202 \\
\hline D from 1.5: C & .216 & .217 & .217 & .216 & .208 \\
\hline D from 1.0: C & .204 & .214 & .216 & .221 & .228 \\
\hline D from $0.5: \mathrm{C}$ & .152 & .181 & .190 & .208 & .253 \\
\hline
\end{tabular}

Note. Personality-Criterion relationships refers to the linearity of the Conscientiousness-criterion relationship, where IP: None indicates a linear

relationship and IP 2.0: C, IP 1.5: C, IP 1.0: C, and IP 0.5: C indicate curvilinear relationships with IP = 2.0, 1.5, 1.0, and 0.5, respectively (the

Emotional Stability-criterion relationship is always linear; see Table 2). Selection Operationalization refers to how Conscientiousness scores are used in selecting individuals, where Linear uses the Conscientiousness score itself and D from 2.0: C, D from 1.5: C, D from 1.0: C, and D from 
0.5: $\mathrm{C}$ use the distance between the Conscientiousness score and 2.0, 1.5, 1.0, and 0.5, respectively. For Conscientiousness alone selection, these values alone are used; for Conscientiousness plus Emotional Stability selection, these values plus Emotional Stability scores are used (see Table 4). Within each selection ratio, bold indicates where the selection operationalization and the criterion are conceptually aligned and underline indicates the selection operationalization that yields maximum mean performance for each underlying predictor-criterion relationship. IP $=$ inflection point; $\mathrm{D}=$ distance $\mathrm{C}=$ Conscientiousness.

A couple of general patterns are worth noting initially. First, the pattern of results between the two selection ratios appears sensible. As expected, in general the mean performance of selected individuals is higher when the selection ratio is lower (e.g., in Table 7, the mean performance values are often in the $.300 \mathrm{~s}$ or $.400 \mathrm{~s}$ when the selection ratio is .10 , but in the $.200 \mathrm{~s}$ when the selection ratio is .50). Second, with a few exceptions, mean performance is maximized for a given set of true underlying personality-criterion relationships (i.e., for a given column) when the selection approach used matches those relationships. For instance, focusing on Conscientiousness validity of .20 and selection ratio of .10 (see Table 6), when the true underlying personality-criterion relationships are linear for both predictors (column IP: None), mean performance is maximized when the selection approach corresponds to this situation (i.e., a simple composite of the two predictors is used; row Linear). This correspondence is demonstrated in the tables when the bold number in a given column and selection ratio (reflecting where maximum mean performance should be) is also underlined (reflecting where maximum mean performance is). For many cases, the bold number is in fact also underlined, indicating using a selection process corresponding to the underlying predictor-criterion relationships maximized mean performance. There are, however, some cases where the bold and underlined numbers are not the same, but these seem to involve very minor differences in mean performance (e.g., .283 vs. .288) and selection approaches that are very similar in nature (e.g., D from 2.0: C vs. D from 1.5: C). These cases thus appear to reflect minor differences where similar selection approaches produce similar mean performance. Note also that these cases appear to be more common for Conscientiousness alone selection when validity is relatively low (.10; see Table 5). It may be that the precise selection variable operationalization matters less when using a single low-validity predictor. Finally, it should also be noted that, although the bold numbers reflect cases where the underlying relationships and selection variables are conceptually aligned, these selection variables are calculated using an approach that does not completely match the data-generation process (e.g., several selection variables are unit-weighted sums involving distances from an inflection point, rather than regression-weighted sums involving squared terms). This imperfect matching is common in practice but creates the potential for less than ideal results even when the selection composite is conceptually appropriate.

Of most interest are the mean performance decrements associated with varying types and levels of mismatch between the selection approach and the true underlying personality-criterion relationships. These decrements reflect the negative consequences associated with implementing selection procedures inappropriately in terms of linearity and inflection point. Tables 5 to 7 show clear patterns of mean performance decrements as the selection approach moves further away from the true underlying relationships. For instance, for Conscientiousness validity of .30, selection ratio of .10, and Conscientiousness alone selection, when the Conscientiousnesscriterion relationship is linear, mean performance goes from .515 (most appropriate selection approach) down to .151 (least appropriate selection approach) for a difference of .364. Similarly, in the same condition, when the Conscientiousness-criterion relationship is curvilinear with an inflection point of 0.5 , mean performance goes from .335 (most appropriate selection approach) to -.099 (least appropriate selection approach) for a difference of .434 (see Table 7). These differences would likely be practically meaningful in many selection situations, as both involve 
losses of approximately .40 SDs where mean performance of selected individuals goes from clearly above average to about average.

However, the magnitude of these decrements depends on the nature of the mismatch, the selection ratio, and the number of predictors used in selection. It is clear, for example, that incorrectly implementing a selection approach either way in terms of linearity — assuming a curvilinear relationship when it is in fact linear or assuming a linear relationship when it is in fact curvilinear - can have detrimental effects. However, the tables also highlight a potentially important trend: With high levels of selectivity (low selection ratios), assuming linear relationships when they are actually curvilinear can be somewhat more detrimental than assuming curvilinear relationships when they are actually linear. This is sensible because when there is a low selection ratio and an inverted-U-shaped relationship, selecting based on a linear assumption (i.e., selecting those high on the personality characteristic) involves hiring those who are on the downward slope of the relationship and thus relatively low in performance. However, with a low selection ratio and a linear relationship, selecting based on a curvilinear assumption (i.e., selecting those moderate on the personality characteristic) involves hiring those who are in the middle of the relationship and thus with average performance.

Tables 5 to 7 also clearly demonstrate that the selection ratio plays an important role in this context. Specifically, being less selective significantly reduces the magnitude of mean performance decrements associated with incorrectly implementing the selection approach. As shown in Tables 5 to 7 , when the selection ratio is .50, mismatched selection approaches generally produce small decreases in mean performance. Some decrements occur in a few cases, but generally only when the selection approach is substantially different from the underlying relationships. Thus, at least in the range of situations simulated, a mismatched selection approach appears to be much less of an issue when the selection ratio is relatively large and conversely, is more of a problem in an organization that is more selective.

Finally, the tables also demonstrate that mean performance decrements tend to be somewhat smaller for Conscientiousness plus Emotional Stability selection than for Conscientiousness alone selection. This also makes sense in that it indicates the negative effects associated with incorrectly specifying the linearity or inflection point for one predictor can be mitigated to some degree by including a correctly modeled additional predictor.

\section{Discussion}

Personality measures continue to receive extensive research attention in selection settings, with numerous studies indicating these assessments predict important dimensions of job performance. As a result, personality currently plays a prominent role in selection research and practice (e.g., Hough \& Oswald, 2008; Oswald \& Hough, 2010). Despite this, the precise form of many personality-performance relationships remains unclear, as both conceptual considerations and empirical evidence regarding linearity are mixed (e.g., Benson \& Campbell, 2007; Day \& Silverman, 1989; LaHuis et al., 2005; Le et al., 2010; Robie \& Ryan, 1999). This means it is quite likely that selection procedures involving personality measures are often implemented in less than optimal ways, where the manner in which selection decisions are made may not match the underlying relationships as closely as might be done if relationships were understood or explored a bit more. The present simulation study examined this fundamental problem by quantifying mean performance decrements associated with varying degrees of mismatch between the selection approach used and the underlying personality-performance relationships that were 
modeled. The goal was to highlight the circumstances where this issue might lead to particular benefits for selection researchers and practitioners.

Results indicated that improperly specifying selection procedures in terms of linearity and inflection point can have noteworthy negative effects on the mean performance of those selected. For instance, when the selection ratio was .10, there were clear decrements in mean performance when a linear Conscientiousness-criterion relationship existed but selection procedures assumed a nonlinear relationship (inflection point $=0.5$ ), and when a nonlinear Conscientiousness-criterion relationship existed (inflection point $=0.5$ ) but selection procedures assumed a linear relationship (see Tables 5-7). These results also indicated implementing selection assuming linear relationships when they are actually curvilinear could be more detrimental than implementing selection assuming curvilinear relationships when they are actually linear. This pattern stems from the fact that when predictor-criterion relationships are nonlinear with relatively low inflection points, high predictor scores are associated with low performance. Thus, a selection approach that does not take this into account - and instead is implemented such that high predictor scores increase the likelihood of selection-results in a large number of low performers being hired. Therefore, in some circumstances, failing to identify nonlinear relationships may be more detrimental than assuming nonlinearity where it does not exist. This finding highlights the importance of further research on the conditions under which curvilinear relationships are more likely to be found (e.g., Le et al., 2010).

However, the magnitude of these mean performance decrements also varied based on (a) the extent of the mismatch between the selection approach and the underlying relationships, (b) the selection ratio, and (c) the number of predictors used. The examples previously provided represent decrements when this mismatch is relatively large (at least given the conditions simulated in this study), where the Conscientiousness-criterion relationship is assumed to be nonlinear with a moderately low inflection point when it is in fact linear or vice versa. In cases where the mismatch is smaller (e.g., assuming a relatively high inflection point when the relationship is linear), mean performance decrements may be less of a concern. In addition, the decrements in mean performance associated with a lack of fit between the selection approach and the underlying relationships tended to be smaller at higher selection ratios. This pattern likely reflects the fact that, when a larger proportion of applicants are hired, the selected group contains a relatively wide range of predictor scores. As a result, the precise manner in which selection decisions are made is less important, as long as the selection approach is not completely inappropriate (e.g., selecting at the extremes - highest and lowest scores-when there is an inverted-U-shaped relationship). This may have important practical implications, because it indicates this issue of correctly identifying the form of personality-performance relationships is less critical when selection ratios are relatively large (e.g., near .50) than when they are relatively small (e.g., near .10). Finally, performance decrements also tended to be smaller when Conscientiousness and Emotional Stability were used than when Conscientiousness alone was used. This finding may also have practical value in that it suggests this issue may be less of a concern when additional correctly modeled predictors can be included in the selection system.

Although the current study has begun to address the problem of negative consequences associated with incorrectly implementing personality-based selection approaches, several issues require additional research. First, this study examined several scenarios in terms of the form of the relationships and the selection approaches used, but additional conditions should be investigated. Future studies might examine other nonlinear relationships (e.g., varying $R^{2}$ for the linear and nonlinear terms), relationships that incorporate group differences (e.g., implications of 
nonlinearity or differential nonlinearity for adverse impact), and alternative selection approaches (e.g., combining general mental ability and Conscientiousness to explore implications of curvilinearity in this scenario; see Schmidt \& Hunter, 1998). A particularly useful direction may be examining asymptotic relationships, as the current study focused on quadratic relationships. Second, this simulation work also clearly highlights the need for more empirical studies on the form of personality-performance relationships. The current results demonstrate that in some situations inappropriate selection approaches can have notable negative consequences. Given this, more work is needed to help in identifying when personality-performance relationships are likely to be linear versus nonlinear to inform selection implementation decisions. Finally, the current study examined performance-related outcomes that have been the focus of previous research, but alternative criteria might also be relevant in the context of curvilinear relationships with personality (e.g., turnover, where extreme levels of personality may lead to higher turnover). Expanding this research to different criteria might illuminate more widely the nature and prevalence of nonlinear relationships with personality predictors. 
References

1. Aiken, L. S. and West, S. G. 1991. Multiple regression: Testing and interpreting interactions, Thousand Oaks, CA: Sage.

2. Arthur, W. Jr., Woehr, D. J. and Graziano, W. G. 2001. Personality testing in employment settings: Problems and issues in the application of typical selection practices. Personnel Review, 30: 657-676. doi:10.1108/EUM0000000005978

3. Barrick, M. R. and Mount, M. K. 1991. The Big Five personality dimensions and job performance: A meta-analysis. Personnel Psychology, 44: 1-26. doi:10.1111/j.17446570.1991.tb00688.x

4. Barrick, M. R., Mount, M. K. and Judge, T. A. 2001. Personality and performance at the beginning of the new millennium: What do we know and where do we go next?. International Journal of Selection and Assessment, 9: 9-30. doi:10.1111/1468-2389.00160

5. Benson, M. J. and Campbell, J. P. 2007. To be, or not to be, linear: An expanded representation of personality and its relationship to leadership performance. International Journal of Selection and Assessment, 15: 232-249. doi:10.1111/j.1468-2389.2007.00384.x

6. Connelly, B. S., \& Ones, D. S. 2010. An other perspective on personality: Meta-analytic integration of observers' accuracy and predictive validity. Psychological Bulletin, 136, 10921122 doi: http://dx.doi.org/10.1037/a0021212

7. Coward, W. M. and Sackett, P. R. 1990. Linearity of ability-performance relationships: A reconfirmation. Journal of Applied Psychology, 75: 297-300. doi:10.1037/0021-9010.75.3.297

8. Cucina, J. M. and Vasilopoulos, N. L. 2005. Nonlinear personality-performance relationships and the spurious moderating effects of traitedness. Journal of Personality, 73: 227-259. doi:10.1111/j.1467-6494.2004.00309.x

9. Dawes, R. M. and Corrigan, B. 1974. Linear models in decision making. Psychological Bulletin, 81: 95-106. doi:10.1037/h0037613

10. Day, D. V. and Silverman, S. B. 1989. Personality and job performance: Evidence of incremental validity. Personnel Psychology, 42: 25-36. doi:10.1111/j.1744-6570.1989.tb01549.x

11. Dunnette, M. D. 1963. A modified model for test validation and selection research. Journal of Applied Psychology, 47: 317-323. doi:10.1037/h0047635

12. Grant, A. M. and Schwartz, B. 2011. Too much of a good thing: The challenge and opportunity of the inverted U. Perspectives on Psychological Science, 6: 61-76.

doi:10.1177/1745691610393523 
13. Hogan, J. and Holland, B. 2003. Using theory to evaluate personality and job-performance relations: A socioanalytic perspective. Journal of Applied Psychology, 88: 100-112. doi:10.1037/0021-9010.88.1.100

14. Hough, L. M. and Oswald, F. L. 2008. Personality testing and industrial-organizational psychology: Reflections, progress, and prospects. Industrial and Organizational Psychology: Perspectives on Science and Practice, 1: 272-290. doi:10.1111/j.1754-9434.2008.00048.x

15. Hurtz, G. M. and Donovan, J. J. 2000. Personality and job performance: The Big Five revisited. Journal of Applied Psychology, 85: 869-879. doi:10.1037/0021-9010.85.6.869

16. Judge, T. A., Bono, J. E., Ilies, R. and Gerhardt, M. W. 2002. Personality and leadership: A qualitative and quantitative review. Journal of Applied Psychology, 87: 765-780.

doi:10.1037/0021-9010.87.4.765

17. LaHuis, D. M., Martin, N. R. and Avis, J. M. 2005. Investigating nonlinear conscientiousness-job performance relations for clerical employees. Human Performance, 18: 199-212. doi:10.1207/s15327043hup1803_1

18. Le, H., Oh, I., Robbins, S. B., Ilies, R., Holland, E. and Westrick, P. 2010. Too much of a good thing: Curvilinear relationships between personality traits and job performance. Journal of Applied Psychology, 96: 113-133. doi:10.1037/a0021016

19. Markon, K. E., Krueger, R. F. and Watson, D. 2005. Delineating the structure of normal and abnormal personality: An integrative hierarchical approach. Journal of Personality and Social Psychology, 88: 139-157. doi:10.1037/0022-3514.88.1.139

20. Murphy, K. R. 1996. "Individual differences in behavior in organizations: Much more than g”. In Individual differences and behavior in organizations, Edited by: Murphy, K. R. 3-30. San Francisco, CA: Jossey-Bass.

21. Nettle, D. 2006. The evolution of personality variation in humans and other animals. American Psychologist, 61: 622-631. doi:10.1037/0003-066X.61.6.622

22. Oh, I., Wang, G., \& Mount, M. K.2011Validity of observer ratings of the five-factor model of personality traits: A meta-analysis. Journal of Applied Psychology, 96, 762-773 doi:http://dx.doi.org/10.1037/a0021832

23. Ones, D. S., Dilchert, S., Viswesvaran, C. and Judge, T. A. 2007. In support of personality assessment in organizational settings. Personnel Psychology, 60: 995-1027. doi:10.1111/j.17446570.2007.00099.x

24. Oswald, F. L., Hough, L. M. and Zedeck, S. 2010. "Personality and its assessment in organizations: Theoretical and empirical developments". In APA handbook of industrial and organizational psychology, Vol 2: Selecting and developing members for the organization, 153184. Washington, DC: American Psychological Association. doi:10.1037/12170-005 
25. Robie, C. and Ryan, A. M. 1999. Effects of nonlinearity and heteroscedasticity on the validity of conscientiousness in predicting overall job performance. International Journal of Selection and Assessment, 7: 157-169. doi:10.1111/1468-2389.00115

26. Sackett, P. R. and Lievens, F. 2008. Personnel selection. Annual Review of Psychology, 59: 419-450. doi:10.1146/annurev.psych.59.103006.093716

27. Salgado, J. F. 1997. The five factor model of personality and job performance in the European community. Journal of Applied Psychology, 82: 30-43. doi:10.1037/00219010.82.1.30

28. Schmidt, F. L. and Hunter, J. E. 1998. The validity and utility of selection methods in personnel psychology: Practical and theoretical implications of 85 years of research findings. Psychological Bulletin, 124: 262-274. doi:10.1037/0033-2909.124.2.262

29. Shepperd, J. A. 1991. Cautions in assessing spurious "moderator effects.". Psychological Bulletin, 110: 315-317. doi:10.1037/0033-2909.110.2.315

30. Tett, R. P. and Christiansen, N. D. 2007. Personality tests at the crossroads: A response to Morgeson, Campion, Dipboye, Hollenbeck, Murphy, and Schmitt (2007). Personnel Psychology, 60: 967-993. doi:10.1111/j.1744-6570.2007.00098.x

31. van der Linden, D., Nijenhuis, J. te and Bakker, A. B. 2010. The general factor of personality: A meta-analysis of Big Five intercorrelations and a criterion-related validity study. Journal of Research in Personality, 44: 315-327. doi:10.1016/j.jrp.2010.03.003

32. Vasilopoulos, N. L., Cucina, J. M., \& Hunter, A. E.2007Personality and training proficiency: Issues of bandwidth-fidelity and curvilinearity. Journal of Occupational and Organizational Psychology, 80, 109-131 doi: http://dx.doi.org/10.1348/096317906X102114

33. Whetzel, D. L., McDaniel, M. A., Yost, A. P., \& Kim, N.2010Linearity of personalityperformance relationships: A large-scale examination. International Journal of Selection and Assessment, 18, 310-320 doi: http://dx.doi.org/10.1111/j.1468-2389.2010.00514.x

34. Yerkes, R. M. and Dodson, J. D. 1908. The relation of strength of stimulus to rapidity of habit formation. Journal of Comparative Neurology \& Psychology, 18: 459-482.

doi:10.1002/cne.920180503 